$\xi=-1$

\title{
Power Quality Disturbances Classification using Discrete Wavelet Transform and Support Vector Machine
}

\author{
Tiagrajah V.Janahiraman*, Muhammad Hazwan Harun \\ Centre for Signal Processing and Control Systems, Dept of Electronics and Communication Engineering, College of Engineering, \\ Universiti Tenaga Nasional, Jalan IKRAM-UNITEN, 43300 Kajang, Selangor, Malaysia \\ *Corresponding authorE-mail: tiagrajah@uniten.edu.my
}

\begin{abstract}
Power utility providers and power industry service providers face a significant challenge in identifying the type of Power Quality Disturbances (PQD) automatically. This paper discusses a method to classify PQD using signal decomposition, statistical analysis and machine learning. Firstly, Discrete Wavelet Transform (DWT) is applied on the generated PQD signals to decompose the signal to obtain its representation in time and frequency domain. Secondly, first and second order statistical parameters are computed on the selected subband of DWT. These parameters are used as features vector for the machine learning based classifier. Our database consists of 2400 generated signals of PQD, which were divided into train and test set. Another set of noise corrupted signal database was generated to evaluate the capability of the system. SVM using quadratic kernel was selected as the classifier of the Power Quality Disturbances feature vector. Comparisons were also made with other types of classifiers and other types of mother wavelet filter functions. The results show that the combination of DWT and SVM managed to classify Power Quality Disturbances with high accuracy and has a strong resistance towards noise.
\end{abstract}

Keywords: Power Quality; Machine Learning; Support Vector Machine; Wavelet Transform; Statistical feature

\section{Introduction}

Pure sinusoidal waveforms of voltage and current at $50 \mathrm{~Hz}$ (Malaysia's power line frequency) without any disruptions or defect in waveform at the electrical incoming point is an important aspect of power quality. Currently, there is a rapid growth in production of innovative electronic gadgets and solar power generation appli ances. Certain electronic components may cause electromagnetic disturbances or it can be sensitive to power quality issues, thus, it has heightened the research interest in power quality [1]. Power Quality Disturbances (PQD) is a term that is characterized by the power load that causes variations, randomness and failures in power grid. Some examples of PQD are sag, swell, interruptions, harmonic and flicker [2]. PQD may cause failure or disoperation of end-use equipment [3].

There are also hybrid PQD which consists of two PQD present simultaneously. Example of hybrid PQD are flicker with interruptions, sag with interruptions, and flicker with sag wave. The specification of period and change of amplitude of the voltage signal with respect to its PQD are stated in IEEE 1159 Standards [1]. The cause of PQD can be due to start or stop of large load in an electrical system [4], lightning [5], electric arc furnaces and arc welder in a weak power system [6]. If PQD are not eliminated, it can cause severe damage which leads to failures or breakdown of loads that are sensitive in power systems.

Feature extraction through signal transforms and statistical analysis with pattern recognition using machine learning methods are the most important steps in classifying PQD [7]. An example of widely used feature extraction tool in digital signal processing is Discrete Wavelet Transform (DWT), which was applied by Zhao et al [8], for decomposing the PQD signal into 8 layers using
Daubechies 4 as the mother wavelet function. Chang et al [9] introduced a hybrid approach using DWT with Discrete Fourier Transform (DFT) for extracting salient features from PQD. The Discrete Wavelet trans- form was used to extract a set of features from PQD while Discrete Fourier Transform was used to extract another set of features.

Hilbert Huang Transform was introduced by Saeed et al [10] as a tool to extract the features from PQD. This signal analysis algorithm decomposes the signal into Intrinsic Mode Functions which provide the user with amplitude and frequency data. In [7], the authors introduced modified s-transform to extract features from PQD. In the modified S-transform, 2 adjustable variables were introduced to control the Gaussian window width, which overcome the limitation of time resolution in the standard s-transform. In the authors' work, these features were classified using Back Propagation Neural Network and Support Vector Machine (SVM). Other examples of features extraction method that were reported by other researchers are S-transform [7] and Short Time Fourier Transform [11].

In order to classify the PQD, researchers have used several types of machine learning methods such as Decision Tree [12], SVM [8], Probabilistic Neural Network (PNN) [2], Ensembles method [13] and Artificial Neural Network [14]. Mohanty et al [15] utilized SVM to classify the PQD, which has better generalization when compared to conventional classifier. This classifier can handle very large feature space. SVM is suitable for binary and multiclass automatic classification problems like protein classification, fault in power transformer, and PQD. In [8], SVM was improved by using Particle Swarm Optimization (PSO) to find the optimal tunable parameters, $(\mathrm{C}, \gamma)$, to improve the classification accuracy. Extreme Learning Machine was used by Zhang Et al [7] to classify the PQD features. This classifier has the advantage of simpler 
structure and fast training approach which makes it suitable for rapid development. In [2], classification performance of PNN was improved by introducing Artificial Bee Colony ( $\mathrm{ABC}$ ), which is used as feature selector. The crucial parameter in PNN, which is the spread constant, and most salient features in a large dataset is determined using $\mathrm{ABC}$.

This paper discusses the usage of Discrete Wavelet Transform (DWT) for PQD signal decomposition which is detailed in Section 2. Section 3 details the statistical analysis that was performed on selected sub- band of WT. The classification of PQD using SVM is detailed in Section 4. Section 5 discusses the simulated results and the paper is concluded in 6 .

\section{Wavelet Transform}

Similar to Fourier Transform, Wavelet Transform has the ability to analyse a stationary signal and decompose into different scales and levels of resolution by dilating a type of mathematical function. An example of these level of decomposition can be seen in Figure 5.

Fourier transform has the ability to describe a signal in time domain as a global representation, whereas wavelet transform is capable of extracting local representation in time and frequency domain for a given signal. These characteristics are vital for extracting distinct and high discriminatory features from PQD in time and frequency domain where disturbance transitions are well hidden within PQD events [16]. The Continuous Wavelet Transform (CWT) for a given signal in terms of mother wavelet function, $\psi$, is given in equation 1 .

$$
C W T(m, n)=\frac{1}{\sqrt{m}} \int_{-\infty}^{\infty} f(t) \psi\left(\frac{t-n}{m}\right) d t, m, n \in R, m /=0
$$

Constants $\mathrm{m}$ and $\mathrm{n}$ represent scale and translation parameters in Equation 1. The term $\mathrm{m}$, which is the scale parameter, represents the length of wavelet and oscillatory frequency. Term $\mathrm{n}$ represents the shifting location. However, for digital computing analysis some redundant information are contained within CWT, thus, DWT, represented in equation 2 , is more preferable when compared to CWT [2] [17] [16].

$$
D W T=\frac{1}{\sqrt{a_{0}^{m}}} \sum f(k) \psi\left(\frac{n-k b_{0} a_{0}^{m}}{a_{0}^{m}}\right)
$$

In DWT applications, determination of suitable mother wavelet function plays an important role as every application may require different types of function. Haar, Morlet Dmey, Daubachies, Coiflets and Mexican are some of the types of mother wavelet functions that were used by other researcher to analyze PQD [2]. Figure 1 and Figure 2 shows mother wavelets or $\psi$ of Dmey and Daubechies 4 respectively obtained from MATLAB. In this paper, Daubachies 6 was selected as the mother wavelet function.

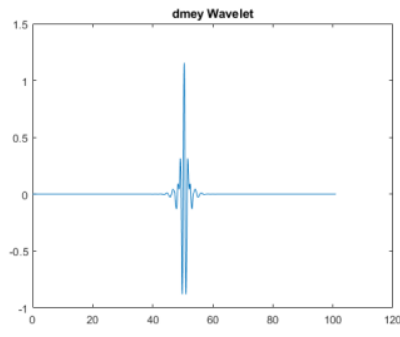

Fig 1: Dmey mother wavelet

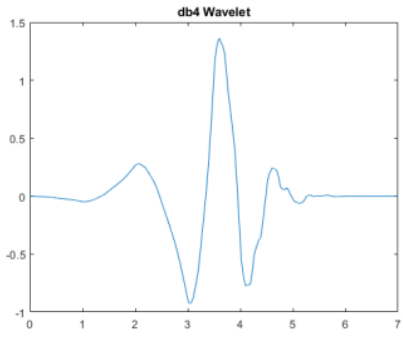

Fig 2: Daubechies 4 mother wavelet

Figure 3 shows the wavelet decomposition flow. The original waveform will be decomposed into decomposition of level 1 and approximation of level 1 using Equation 2. The approximation of level 1 will be decomposed into approximation of level 2 and decomposition of level 2. The process will be repeated until the desired level. An example of Daubechies 4 level 4 decomposition of normal waveform is shown in Figure 4.

\section{Statistical Feature Extraction on Discrete Wavelet transform}

From the decomposed sub-band, first order and second order statistical features listed in Table 1 were computed. Then, all the computed data were normalized based on type of features.

\section{Support Vector Machine}

Support Vector Machine(SVM) is a suitable tool that can be used to classify dataset with large number of samples and features using its associated learning algorithms. By introducing the kernel functions, SVM can achieve the non-linear classification[8] [15]. Samples in the transmission space will be transformed into higher dimensional space, which can be easily separated linearly. This transformation is calculated using kernel functions. The construction of optimal hyperplane in the transformed higher dimensional space with maximum margin between positive and negative samples is achieved using SVM.

Let the training dataset, $x_{i}$, consisting of $m$ samples with corresponding label vector, $y \in\{+1,-1\}$ (i.e. +1 for class 1 and -1 for class 2) to represented as equation 3 .

$D=\left\{(x, y), x_{i} \in R^{n}, y \in\{+1,-1\}, i=1,2, . ., m\right\}$

For linearly separable data, we can define hyperplane as

$w^{T} x+b=0, \quad w \in R^{n}, b \in R$

Table 1: First and Second Orders Statistical Parameter Formulas

\begin{tabular}{l|l}
\hline Features & Formula \\
\hline Power & $E_{i}=\sum_{\{j=1\}}^{N}\left(\left|A_{i j}\right|^{2},\left|D_{i j}\right|^{2}\right)$ \\
Range & $R G_{i}=\operatorname{Max}\left(A_{i j}, D_{i j}\right)-\operatorname{Min}\left(A-i j, D_{i j}\right)$
\end{tabular}




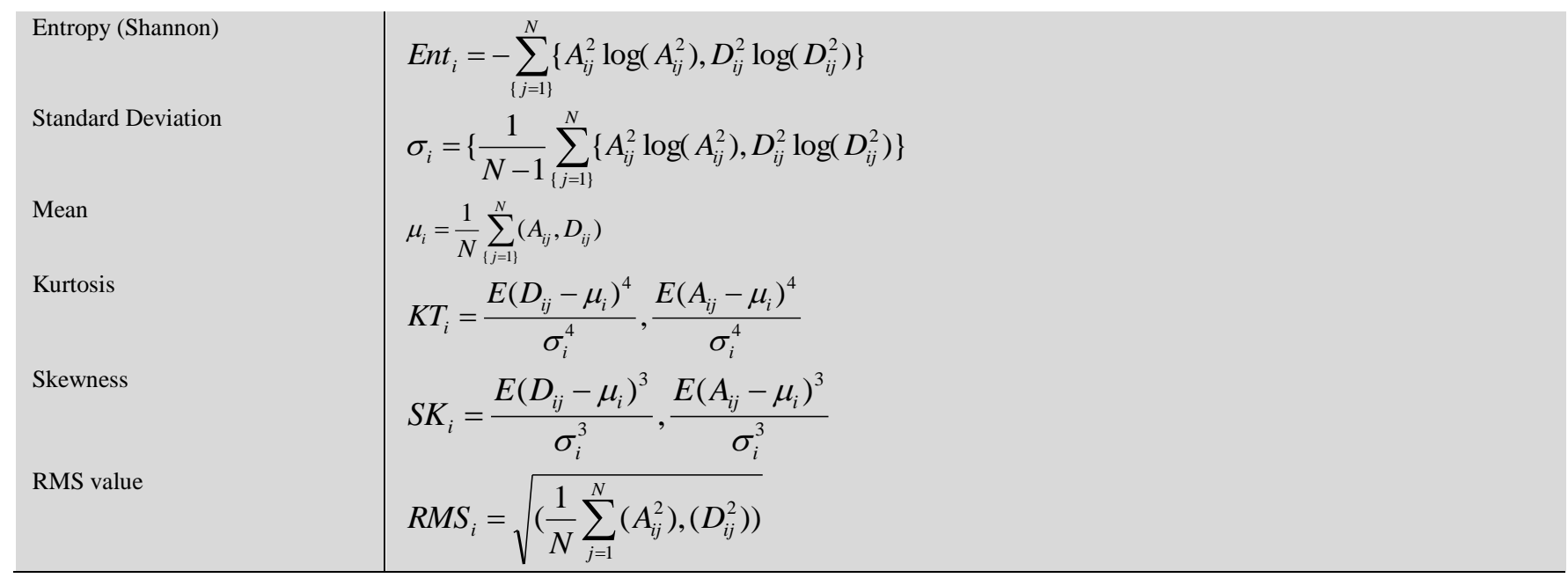

Original Wave Form

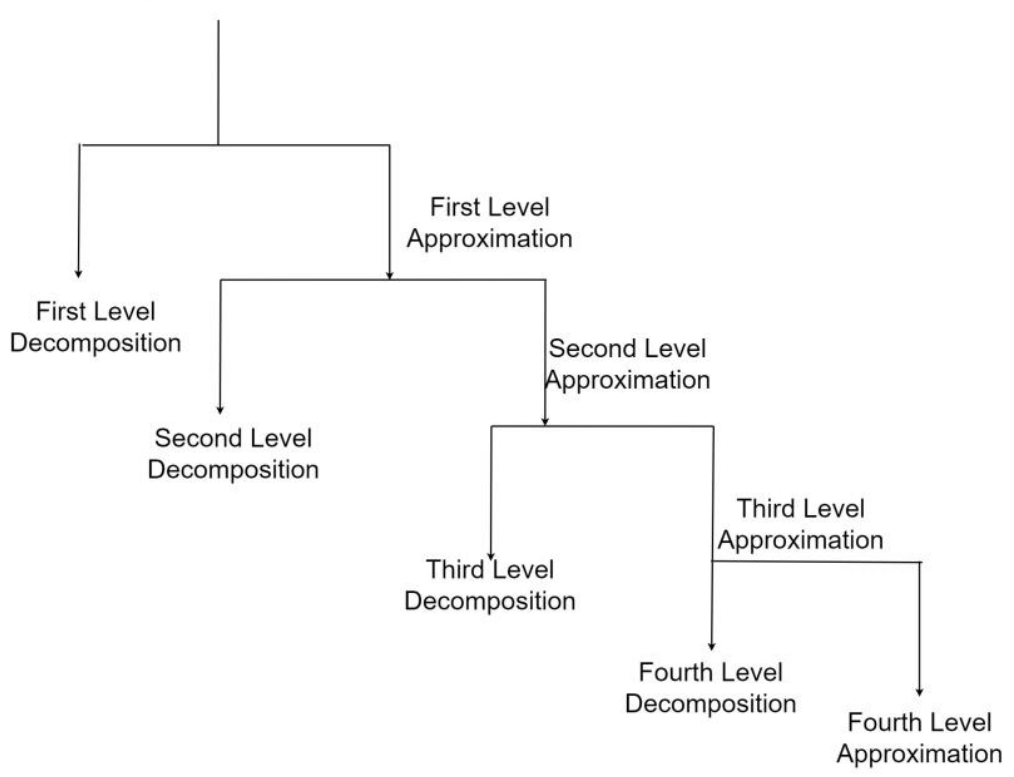

Fig 3: Wavelet Transform Decomposition Chart

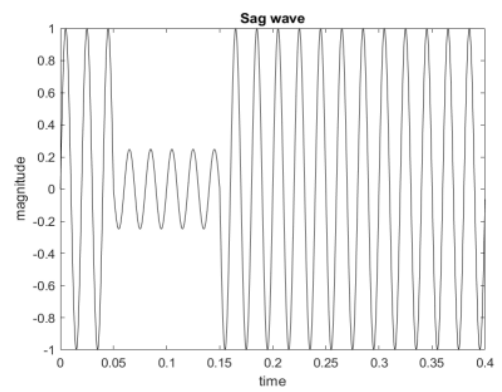

(a) Sag Wave

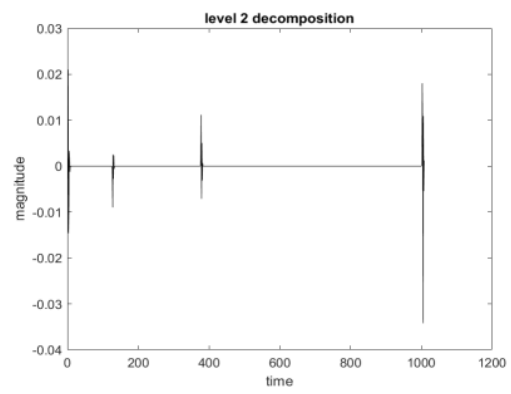

(c) Second Level Decomposition

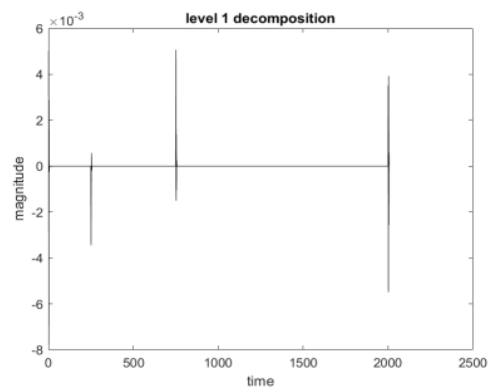

(b) First Level Decomposition

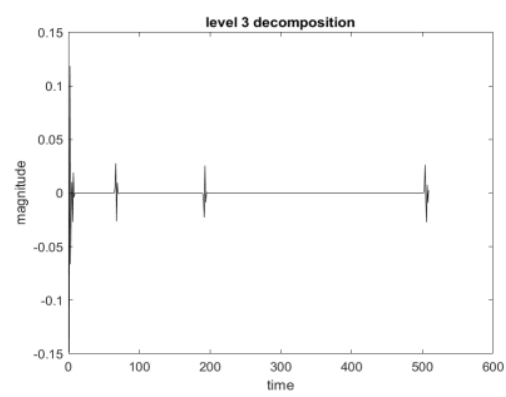

(d) Third Level Decomposition 


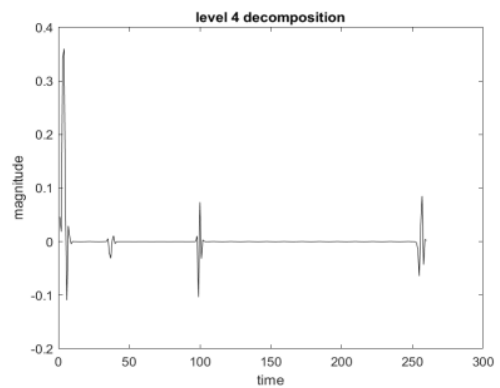

(e) Fourth Level Decomposition

Fig 4: Wavelet Decompositions
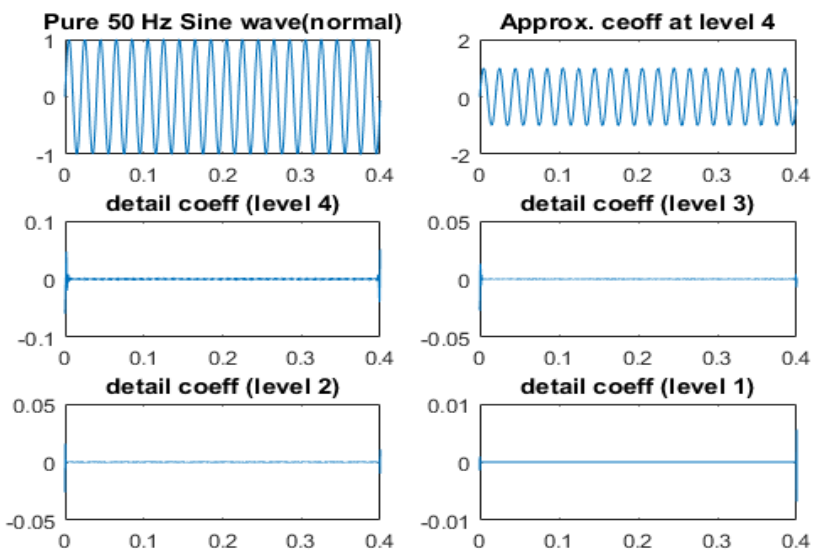

Fig 5: Discrete Wavelet decomposition waveform

here $w$ is an $n$-dimension vector and $b$ is a constant. The decision position for the hyperplane which gives separation in the higher dimensional space is determined by the values $w$ and $b$.

Equation 5 states the condition that must be fulfilled in order to obtain an hyperplane with maximum margin for separating the training vector.

$y_{i}\left(w^{T} x_{i}+b\right) \geq 1 \quad i=1,2, \ldots m$

The distance between the positive and negative samples in the corresponding high dimensional space can be calculated using equation 6 .

$d=\frac{\left|w^{T} x+b\right|}{\|w\|}$

Thus, construction of optimal classification hyper-plane is converted to solve the maximum of the classification interval, namely the minimization problem of $\|\mathrm{w}\|$.

$$
\left\{\begin{array}{l}
\min \frac{1}{2} w^{T} w \\
\operatorname{sty}_{i}\left(w^{T} x_{i}+b\right)-1 \geq 0
\end{array}\right\}
$$

where $i=1,2, \ldots, m$.

To solve for the optimal hyperplane, equation 7 can converted into quadratic programming due to the adoption of Lagrange algorithm and inclusion on Lagrange multipliers, $\alpha=\alpha 1, \ldots, \alpha_{n}$. Support vectors are samples which appears in the interval of separated planes, represented as $x_{i}$. Thus, $w=\sum i \alpha_{i} y_{i} x_{i}$, can be expressed by equation 8 as a classification function.

$$
f(x)=\operatorname{sgn}\left(\sum_{i} a_{i} y_{i} x_{i}, x_{i}\right)
$$

The objective function can be summarized as in equation 9 which deals with non-separable data in the input space.

$$
\min \phi(w, \xi)=\frac{1}{2}\|w\|^{2}+c\left(\sum_{i=1}^{n} \phi\right)
$$

where $\zeta$ is a slack variable and $\mathrm{C}$ is a penalty factor.

A non-linear transformation, $\phi($.$) , optimal separating hyperspace$ can be solved by mapping the input space into features space which is a higher dimensional space [18]. Equation 10 represent the inner product computation.

$$
K\left(x_{i}, x_{j}\right)=\phi\left(x_{i}\right) \cdot \phi\left(x_{j}\right)
$$

where the inner product in Hilbert function can be represented as kernel function, $K\left(x_{i}, x_{j}\right)$. An example of kernel function is the polynomial kernel which is defined in equation 11 for degree-d polynomial.

$K\left(x_{i}, x_{j}\right)=\left(x^{T} y+c\right)^{d}$

The quadratic kernel, by substituting $d=2$ in equation 11 , was used in this paper. Thus, equation 12 summarizes the final classification function.

$f(x)=S G N\left(\sum_{i} a_{i} y_{i} K\left(x_{i}, x_{j}\right) \cdot x+b\right)$

\section{Simulation and discussion}

Based on the noise equations given in Table 2, which were obtained from [2], 12 PQD were generated and used as train dataset. The train dataset consist of 200 samples for each PQD, making it a total of 2400 training samples produced for the simulation. Figures 6 to 12 shows the plot of PQD waveforms. 
These train dataset is then processed using Daubechies 6 mother wavelet function based WT to obtain the level 8 decomposition sub-band. The general flow chart of the simulation is given in Figure 13.

Table 2: First and Second Orders Statistical Parameter Formulas

\begin{tabular}{|c|c|c|c|}
\hline Label & $\begin{array}{l}\text { Power Qual- } \\
\text { ity Disturb- } \\
\text { ances }\end{array}$ & Mathematical equations & Parameters \\
\hline $\mathrm{C} 1$ & Normal & $\begin{array}{l}y(t)=A\left[1 \pm \alpha\left(u\left(t-t_{1}\right)-\right.\right. \\
\left.\left.u\left(t-t_{2}\right)\right)\right] \sin (\omega t)\end{array}$ & $\begin{array}{c}\alpha \leq 0.1 ; T \leq t_{2}-t_{1} \\
\leq 9 T\end{array}$ \\
\hline $\mathrm{C} 2$ & Sag & $\begin{array}{l}y(t)=A\left[1-\alpha\left(u\left(t-t_{1}\right)-\right.\right. \\
\left.\left.u\left(t-t_{2}\right)\right)\right] \sin (\omega t)\end{array}$ & $\begin{array}{c}0.1 \leq \alpha \leq 0.9 ; T \leq t_{2} \\
-t_{1} \leq 9 T\end{array}$ \\
\hline C3 & Swell & $\begin{array}{l}y(t)=A\left[1+\alpha\left(u\left(t-t_{1}\right)-\right.\right. \\
\left.\left.u\left(t-t_{2}\right)\right)\right] \sin (\omega t)\end{array}$ & $\begin{array}{c}0.1 \leq \alpha \leq 0.8 ; T \leq t_{2} \\
-t_{1} \leq 9 T\end{array}$ \\
\hline $\mathrm{C} 4$ & Interruption & $\begin{array}{l}y(t)=A\left[1-\alpha\left(u\left(t-t_{l}\right)-\right.\right. \\
\left.\left.u\left(t-t_{2}\right)\right)\right] \sin (\omega t)\end{array}$ & $\begin{array}{c}0.9 \leq \alpha \leq 1.0 ; T \leq t_{2} \\
-t_{1} \leq 9 T\end{array}$ \\
\hline C5 & Harmonics & $\begin{array}{l}y(t)=A\left[\alpha_{1} \sin (\omega t)+\alpha_{3}\right. \\
\sin (3 \omega t)+\alpha_{5} \sin (5 \omega t)+ \\
\left.\alpha_{7} \sin (7 \omega t)\right]\end{array}$ & $\begin{array}{c}0.05 \leq \alpha_{3} \alpha_{5} \leq 0.15 \\
\sum \alpha_{i}^{2}=1\end{array}$ \\
\hline C6 & $\begin{array}{l}\text { Sag with } \\
\text { harmonics }\end{array}$ & $\begin{array}{l}y(t)=A\left[1-\alpha\left(u\left(t-t_{l}\right)-\right.\right. \\
\left.\left.u\left(t-t_{2}\right)\right)\right]\left[\alpha_{1} \sin (\omega t)+\alpha_{3}\right. \\
\left.\sin (3 \omega t)+\alpha_{5} \sin (5 \omega t)\right]\end{array}$ & $\begin{array}{c}0.1 \leq \alpha \leq 0.9 ; T \leq t_{2} \\
-t_{1} \leq 9 T \\
0.05 \leq \alpha_{3} \alpha_{5} \leq 0.15 \\
\sum \alpha_{i}^{2}=1\end{array}$ \\
\hline C7 & $\begin{array}{l}\text { Swell with } \\
\text { harmonics }\end{array}$ & $\begin{array}{l}y(t)=A\left[1+\alpha\left(u\left(t-t_{1}\right)-\right.\right. \\
\left.\left.u\left(t-t_{2}\right)\right)\right]\left[\alpha_{1} \sin (\omega t)+\alpha_{3}\right. \\
\left.\sin (3 \omega t)+\alpha_{5} \sin (5 \omega t)\right]\end{array}$ & $\begin{array}{c}0.1 \leq \alpha \leq 0.8 ; T \leq t_{2} \\
-t_{1} \leq 9 T \\
0.05 \leq \alpha_{3} \alpha_{5} \leq 0.15 \\
\sum \alpha_{i}^{2}=1\end{array}$ \\
\hline $\mathrm{C} 8$ & $\begin{array}{l}\text { Interruption } \\
\text { with har- } \\
\text { monics }\end{array}$ & $\begin{array}{l}y(t)=A\left[1-\alpha\left(u\left(t-t_{l}\right)-\right.\right. \\
\left.\left.u\left(t-t_{2}\right)\right)\right]\left[\alpha_{1} \sin (\omega t)+\alpha_{3}\right. \\
\left.\sin (3 \omega t)+\alpha_{5} \sin (5 \omega t)\right]\end{array}$ & $\begin{array}{c}0.9 \leq \alpha \leq 1.0 ; T \leq t_{2} \\
-t_{1} \leq 9 T \\
0.05 \leq \alpha_{3} \alpha_{5} \leq 0.15 \\
\sum \alpha_{i}^{2}=1\end{array}$ \\
\hline C9 & Flicker & $\begin{array}{l}y(t)=A\left[1+\alpha_{f} \sin (\beta \omega t)\right] \\
\sin (\omega t)\end{array}$ & $\begin{array}{c}0.1 \leq \alpha_{f} \leq 0.2 ; 5 \leq \beta \\
\leq 20 \mathrm{~Hz}\end{array}$ \\
\hline $\mathrm{C} 10$ & $\begin{array}{l}\text { Flicker with } \\
\text { harmonics }\end{array}$ & $\begin{array}{l}y(t)=A\left[1+\alpha_{f} \sin (\beta \omega t)\right] \\
\sin (\omega t)\left[\alpha_{I} \sin (\omega t)+\alpha_{3}\right. \\
\left.\sin (3 \omega t)+\alpha_{5} \sin (5 \omega t)\right]\end{array}$ & $\begin{array}{c}0.1 \leq \alpha_{f} \leq 0.2 ; 5 \leq \beta \\
\leq 20 \mathrm{~Hz} \\
0.05 \leq \alpha_{3} \alpha_{5} \leq 0.15 \\
\sum \alpha_{i}^{2}=1\end{array}$ \\
\hline $\mathrm{C} 11$ & $\begin{array}{l}\text { Flicker with } \\
\text { sag }\end{array}$ & $\begin{array}{l}y(t)=A\left[1+\alpha_{f} \sin (\beta \omega t)\right] \\
\sin (\omega t)\left[1-\alpha\left(u\left(t-t_{1}\right)-\right.\right. \\
\left.\left.u\left(t-t_{2}\right)\right)\right]\end{array}$ & $\begin{array}{c}0.1 \leq \alpha_{f} \leq 0.2 ; 5 \leq \beta \\
\leq 20 \mathrm{~Hz} \\
0.1 \leq \alpha \leq 0.9 ; T \leq t_{2} \\
-t_{l} \leq 9 T\end{array}$ \\
\hline $\mathrm{C} 12$ & $\begin{array}{l}\text { Flicker with } \\
\text { swell }\end{array}$ & $\begin{array}{l}y(t)=A\left[1+\alpha_{f} \sin (\beta \omega t)\right] \\
\sin (\omega t)\left[1+\alpha\left(u\left(t-t_{1}\right)-\right.\right. \\
\left.\left.u\left(t-t_{2}\right)\right)\right]\end{array}$ & $\begin{array}{c}0.1 \leq \alpha_{f} \leq 0.2 ; 5 \leq \beta \\
\leq 20 \mathrm{~Hz} \\
0.1 \leq \alpha \leq 0.8 ; T \leq t_{2} \\
-t_{1} \leq 9 T\end{array}$ \\
\hline
\end{tabular}

\subsection{Training Classifier}

The normalized dataset obtained from Table 2 is then used to establish the classification model. To examine the capability of our classification model and to mitigate the problem of overfitting, we utilized the 5 fold cross-validation method. SVM with quadratic kernel was selected in this simulation. The confusion matrix, that allows visualization of the performance of the classification model, was generated from the experimental results is tabulated in Table 3. From the results, it can be observed that the classifier managed to produce an accuracy of $100 \%$ for almost all the PQD except for Harmonics hybrid PQD (harmonics + sag and harmonics + swell). Here, the harmonics with swell PQD is often misclassified as harmonics with sag PQD since they are very similar. The average classification accuracy is $92 \%$.

\section{Conclusion}

This paper presented 12 types of PQD that commonly occur in electricity grid and power systems. In order to classify the PQD, simulated signal were decomposed using DWT. First order and Second order statistical features were computed on the sub-band produced by DWT. The computed statistical parameter were used as features vector for the classifier. This paper shows that the combination of Daubechies 6 WT and SVM have produced a better results in accuracy compared with other combinations of DWT and classifier in classifying PQD even with added noise to the signal.

Table 3: Confusion Matrix (given in \%)

\begin{tabular}{|l|l|l|l|l|l|l|l|l|l|l|l|l|}
\hline & S1 & S2 & S3 & S4 & S5 & S6 & S7 & S8 & S9 & S10 & S11 & S12 \\
\hline S1 & 100 & & & & & & & & & & & \\
\hline S2 & & 100 & & & & & & & & & & \\
\hline S3 & & & 99 & & & & & & & & & \\
\hline S4 & & & 100 & & & & & & & & \\
\hline S5 & & & & & 100 & & & & & & & \\
\hline S6 & & & & & 53 & 48 & & & & & \\
\hline S7 & & & & & & 47 & 52 & & & & & \\
\hline S8 & & & & & & & 100 & & & & \\
\hline S9 & & & & & & & & & 100 & & & \\
\hline S10 & & & & & & & & & 100 & & \\
\hline S11 & & & & & & & & & & & 100 & \\
\hline S12 & & 1 & & & & & & & & & 100 \\
\hline
\end{tabular}

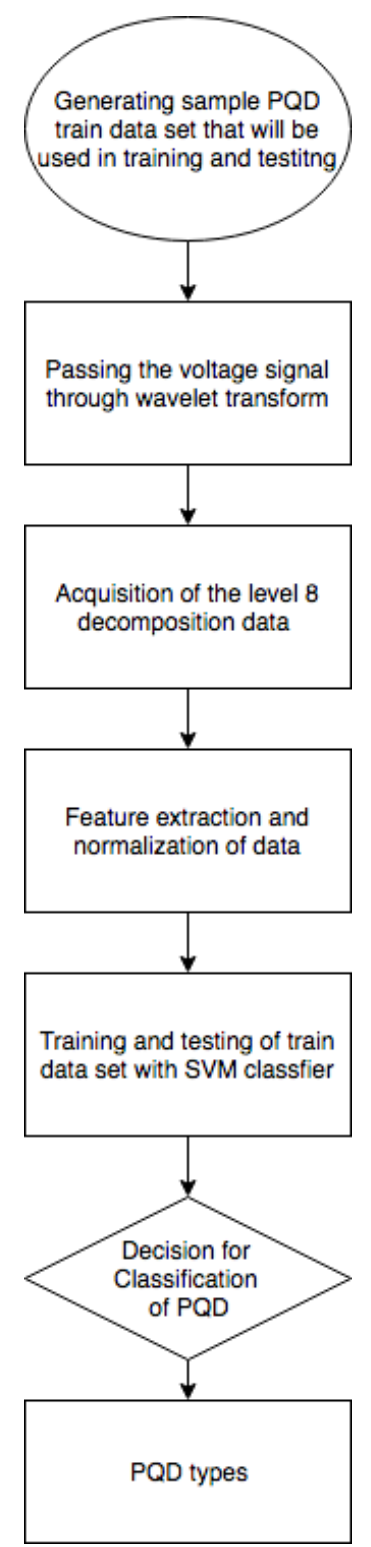

Fig 13: Flow Chart of the simulation 


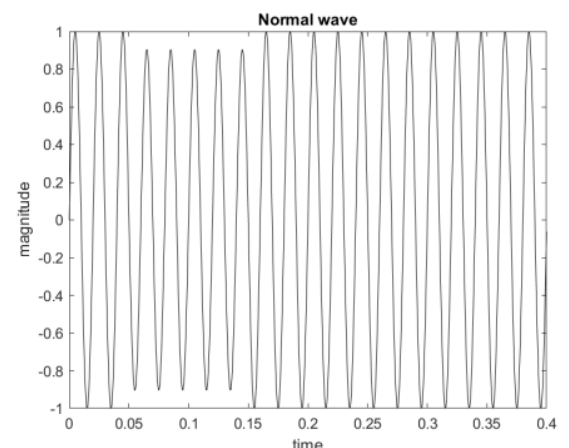

Fig. 6: Normal Wave

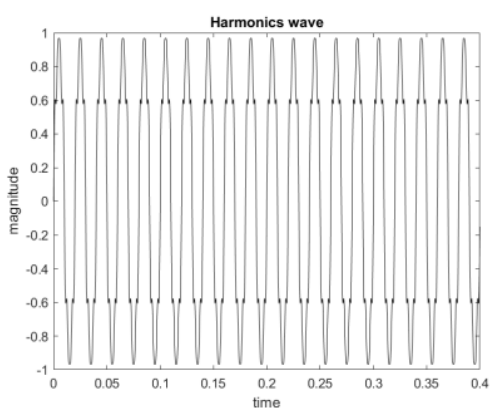

Fig. 7: Harmonics Wave

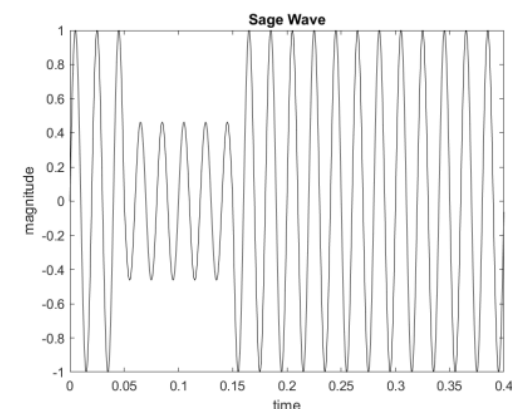

Fig. 8: Sag Wave

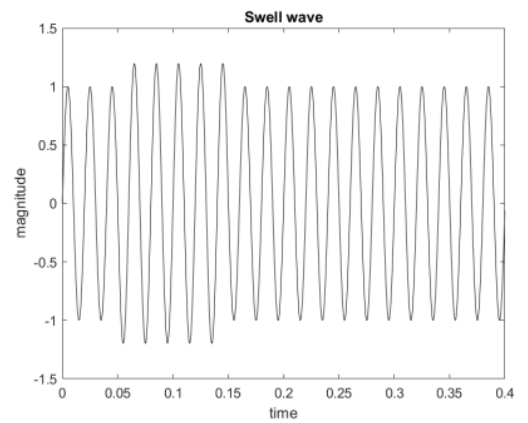

Fig. 9: Swell Wave

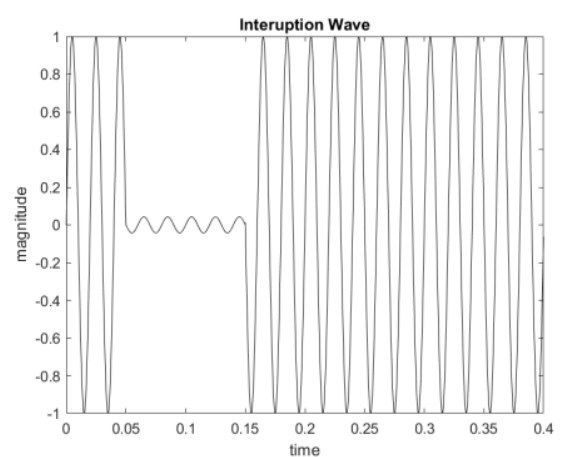

Fig. 10: Interruption Wave

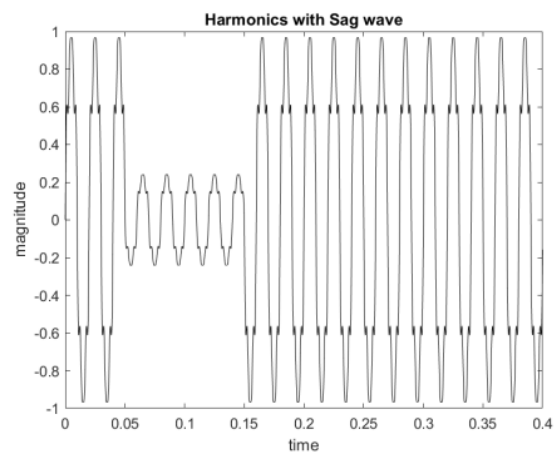

Fig. 11: Harmonics with Sag wave

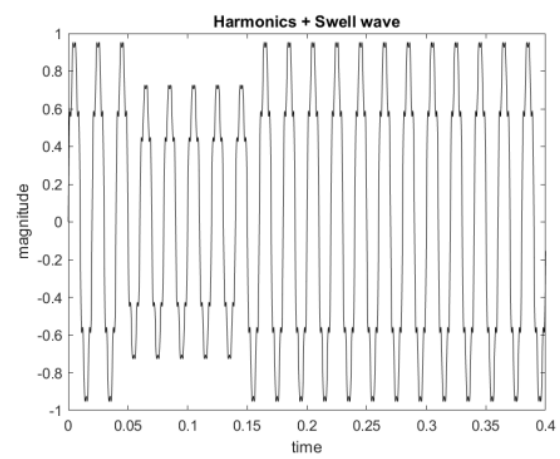

Fig. 11: Harmonics with Swell wave

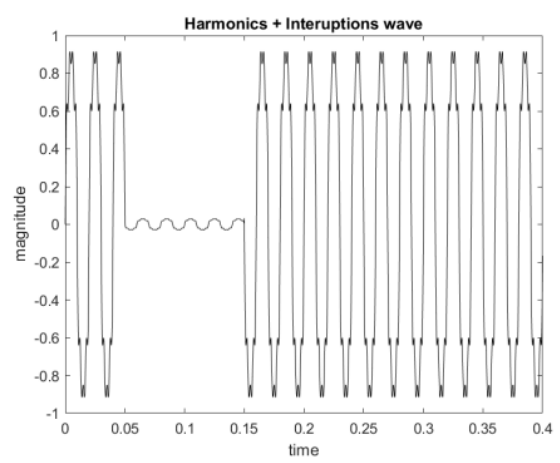

Fig. 12: Harmonics with interruption wave

\section{References}

[1] "Ieee recommended practice for monitoring electric power quality," IEEE Std 1159-2009 (Revision of IEEE Std 1159-1995), pp. c1-81, June 2009.

[2] S. Khokhar, A. A. M. Zin, A. P. Memon, and A. S. Mokhtar, "A new optimal feature selection algorithm for classification of power quality disturbances using discrete wavelet transform and probabilistic neural network," Measurement, vol. 95, pp. 246 - 259, 2017.

[3] N. H. T. Huda, A. R. Abdullah, and M. H. Jopri, "Power quality signals detection using s-transform," in 2013 IEEE 7th International Power Engineering and Optimization Conference (PEOCO), pp. 552-557, June 2013.

[4] A. Domijan, J. T. Heydt, A. P. S. Meliopoulos, M. S. S. Venkata, and S. West, "Directions of research on electric power quality," IEEE Transactions on Power Delivery, vol. 8, pp. 429-436, Jan 1993.

[5] R. Rojin, "A review of power quality problems and solutions in elec- trical power system," International Journal of Advanced Research in Electrical, Electronics and Instrumentation Engineering, vol. 2, no. 11, pp. 5605-5614, 2013.

[6] S. B. Jung, J. C. Kim, K. H. Choi, and G. H. Choe, "A study on flicker evaluation considering power quality disturbance of power system," in 2007 IEEE Power Engineering Society General Meeting, pp. 1-6, June 2007.

[7] S. Zhang, P. Li, L. Zhang, H. Li, W. Jiang, and Y. Hu, "Modified s transform and elm algorithms and their applications in power quality analysis," Neurocomputing, vol. 185, pp. $231-241,2016$. 
[8] Z. Liquan, G. Meijiao, and W. Lin, "Classification of multiple power quality disturbances based on the improved svm," in 2017 Interna- tional Conference on Wireless Communications, Signal Processing and Networking (WiSPNET), pp. 2625-2628, March 2017.

[9] P. C. Chang, G. W. Chang, M. H. Shih, Y. Y. Chen, Y. H. Hong, and Y. K. Yeh, "A hybrid approach for detection and classification of power quality disturbances," in 2017 IEEE Power Energy Society General Meeting, pp. 1-5, July 2017.

[10] S. Alshahrani, M. Abbod, and G. Taylor, "Detection and classification of power quality disturbances based on hilbert-huang transform and feed forward neural networks," in 2016 51st International Universities Power Engineering Conference (UPEC), pp. 1-6, Sept 2016.

[11] P. K. Ray, S. R. Mohanty, N. Kishor, and J. Catalao, "Optimal feature and decision tree based classification of power quality disturbances in distributed generation systems," in 2014 IEEE PES General Meeting Conference Exposition, pp. 1-1, July 2014.

[12] M. Biswal and P. Dash, "Detection and characterization of multiple power quality disturbances with a fast s-transform and decision tree based classifier," Digital Signal Processing, vol. 23, no. 4, pp. 1071 $-1083,2013$.

[13] S. Karasu and S. Bas,kan, "Classification of power quality disturbances by using ensemble technique," in 2016 24th Signal Processing and Communication Application Conference (SIU), pp. 529-532, May 2016.

[14] R. Bhavani and N. R. Prabha, "A hybrid classifier for power quality (pq) problems using wavelets packet transform (wpt) and artificial neural networks (ann)," in 2017 IEEE International Conference on Intelligent Techniques in Control, Optimization and Signal Processing (INCOS), pp. 1-7, March 2017.

[15] S. R. Mohanty, P. K. Ray, N. Kishor, and B. Panigrahi, "Classification of disturbances in hybrid dg system using modular pnn and svm," Inter- national Journal of Electrical Power \& Energy Systems, vol. 44, no. 1, pp. $764-777,2013$.

[16] V. A. Katic' and A. M. Stanisavljevic', "Wavelet transform for voltage dips detection in a microgrid with distributed generation," in 2017 19th European Conference on Power Electronics and Applications (EPE'17 ECCE Europe), pp. P.1-P.10, Sept 2017.

[17] Z. Liu, Q. Zhang, Z. Han, and G. Chen, "A new classification method for transient power quality combining spectral kurtosis with neural network," Neurocomputing, vol. 125, pp. 95 - 101, 2014. Advances in Neural Network Research and Applications Advances in Bio-Inspired Computing: Techniques and Applications.

[18] W. Tong, X. Song, J. Lin, and Z. Zhao, "Detection and classification of power quality disturbances based on wavelet packet decomposition and support vector machines," in 2006 8th international Conference on Signal Processing, vol. 4, 2006. 